\title{
Influence of rotation and sources of nutrients on soil properties and productivity of finger millet (Eleusine coracana L. Gaertn.)
}

\author{
Pavankumar Goudar $^{1 *}$, B. K. Ramachandrappa ${ }^{2}$, M. N. Thimmegowda ${ }^{2}$ and S. Sahoo ${ }^{1}$ \\ ${ }^{1}$ Department of Agronomy, University of Agricultural Sciences, GKVK, Bengaluru-560065 (Karnataka), INDIA \\ ${ }^{2}$ AICRP on Dry Land Agriculture,University of Agricultural Sciences, GKVK, Bengaluru-560065 (Karnataka), \\ INDIA \\ *Corresponding author. E-mail: pavankg77@gmail.com
}

Received: July 15, 2016; Revised received: January 26, 2017; Accepted: April 7, 2017

\begin{abstract}
A field experiment was conducted at the All India Co-ordinated Research Project for Dryland Agriculture, UAS, GKVK, Bengaluru during kharif 2013-14. The experiment was laid out with 20 treatment combinations with three factors using factorial RBD with two replications comprised of on a permanent manurial trial with $35^{\text {th }} \mathrm{crop}$ cycle. Application of FYM at $10 \mathrm{t} \mathrm{ha}^{-1}$ has recorded significantly higher grain yield $\left(1.76 \mathrm{t} \mathrm{ha}^{-1}\right)$, maximum water holding capacity (MWHC) of $43.85 \%$ and dehydrogenase activity (DHA) of $357.60 \mu \mathrm{g}$ TPF g ${ }^{-1} 24 \mathrm{~h}^{-1}$ obtained after harvest of the crop as compared to application of maize residues at $5 \mathrm{t} \mathrm{ha}^{-1}\left(1.37 \mathrm{t} \mathrm{ha}^{-1}, 42.27 \%\right.$ and $193.0 \mu \mathrm{g}$ TPF g $\mathrm{g}^{-1}$ $24 \mathrm{~h}^{-1}$ respectively) due to improved growth and yield parameters of finger millet. However, finger millet-groundnut rotation has given significantly higher grain yield $\left(1.78 \mathrm{t} \mathrm{ha}^{-1}\right)$, MWHC (43.66 \%) and DHA (298.48 $\left.\mu \mathrm{gPF} \mathrm{g}^{-1} 24 \mathrm{~h}^{-1}\right)$ after harvest of the crop over mono cropping of finger millet $\left(1.34 \mathrm{t} \mathrm{ha}^{-1}, 42.46 \%\right.$ and $252.12 \mu \mathrm{HPFg}^{-1} 24$ $\mathrm{h}^{-1}$ respectively ). Among different nutrient sources, application of organic matter with $100 \%$ RDF have given significantly higher grain yield $\left(2.74 \mathrm{t} \mathrm{ha}^{-1}\right)$, MWHC $(45.86 \%)$ and DHA $\left(431.13 \mu \mathrm{g} \mathrm{TPF} \mathrm{g}{ }^{-1} 24 \mathrm{~h}^{-1}\right)$ after harvest of the crop compared to absolute control $\left(0.28 \mathrm{t} \mathrm{ha}^{-1}, 41.76 \%\right.$ and $133.67 \mathrm{\mu g} \mathrm{TPFg}^{-1} 24 \mathrm{~h}^{-1}$ respectively). The $100 \%$ recommended dose of fertilizer + organic matter significantly increased yield attributes because of improved soil physical and chemical properties and increased microbial activity of the soil with continued application of organic matter.
\end{abstract}

Keywords: Enzyme activity, Finger millet, Soil environment, Soil properties

\section{INTRODUCTION}

Nearly seventy per cent of net cultivated area of India falls under rainfed condition, where moisture scarcity due to aberrant weather condition is common. Finger millet suits well in these conditions due to its inherent ability to withstand aberrant weather conditions. Nutrient mining under conventional cropping systems ultimately resulted in deficiencies of micronutrients, reduced soil biological activity at last in to poor yields or complete crop damage in certain areas. With the need to produce more food grains elimination of chemical fertilizers do not come in to picture. In view of maintaining harmony between agricultural productivity and side by side maintaining ecological balance, it is a must to adopt integrated soil building practices for improving and stabilizing soil productivity alone and agricultural sustainability in whole which was observed at Dryland Agriculture Project, Bangalore where significantly higher $\mathrm{pH}$, organic carbon, NPK in soil was observed in crop grown with 10 tonnes of FYM and legume rotation over maize residue under mono cropping (Anonymous, 2013). Yadav et al. (2006) also reported improved soil health through application of FYM in continuous manner. Mono-culture of cereals leads to its adverse effect on soil environment. In rotation system, the nutrient is being recycled which makes it an impeccable pillar of sustainable production system. Inclusion of legumes in a rotation improves soil fertility and consequently the productivity of succeeding crop. The present investigation was carried out with an aim to meet the requirements of sustainable and ecological production technique.

\section{MATERIALS AND METHODS}

The investigation was carried out in the Kharif season of 2013 at the AICRP on Dryland Agriculture, Gandhi Krishi Vignana Kendra (GKVK), University of Agricultural Sciences, Bengaluru. The soil textural class of the experimental site was red sandy clay loam with 0.2 $\mathrm{dS} \mathrm{m} \mathrm{m}^{-1}$ electrical conductivity, $0.45 \%$ organic carbon, $\mathrm{pH}$ of 5.25 and 173,62 and $82 \mathrm{~kg} \mathrm{ha}^{-1}$ of nitrogen, available phosphorus and available potassium, respectively. The study was a part of the long term manurial experiment with $35^{\text {th }}$ crop cycle using Factorial RCBD with three factors with three different levels replicated twice.

The first factor was source of organic matter with two levels $\left\{\mathrm{S}_{1}\right.$ :- FYM $10 \mathrm{t} \mathrm{ha}^{-1}$ and $\mathrm{S}_{2}$ :- maize residue $5 \mathrm{t}$ 
Pavankumar Goudar et al. / J. Appl. \& Nat. Sci. 9 (2): 680 - 687 (2017)

Table 1. Maximum water holding capacity, infiltration rate and bulk density of soil before sowing and after harvest of crop as influenced by legume rotation, use of organic and inorganic sources of nutrients.

\begin{tabular}{|c|c|c|c|c|c|c|c|c|c|c|c|c|c|}
\hline & \multicolumn{2}{|c|}{ M.W.H.C (\%) } & \multicolumn{2}{|c|}{ I.R $\left(\mathrm{cm} \mathrm{hr}^{-1}\right)$} & \multicolumn{2}{|c|}{ B.D (g cc-1) } & & \multicolumn{2}{|c|}{ M.W.H.C (\%) } & \multicolumn{2}{|c|}{ I.R $\left(\mathrm{cm} \mathrm{hr}^{-1}\right)$} & \multicolumn{2}{|c|}{ B.D (g cc-1) } \\
\hline & Before & After & Before & After & Before & After & & Before & After & Before & After & Before & After \\
\hline \multicolumn{7}{|c|}{ Source of organic manure (S) } & \multicolumn{7}{|c|}{ Interaction (RxT) } \\
\hline$\overline{S_{1}}$ & 43.48 & 43.85 & 17.53 & 18.91 & 1.34 & 1.35 & $\mathrm{R}_{1} \mathrm{~T}_{1}$ & 43.19 & 42.05 & 17.18 & 16.6 & 1.39 & 1.4 \\
\hline $\mathrm{S}_{2}$ & 42.37 & 42.27 & 14.03 & 14.44 & 1.42 & 1.42 & $\mathrm{R}_{1} \mathrm{~T}_{2}$ & 42.9 & 43.58 & 18.1 & 16.26 & 1.37 & 1.36 \\
\hline S.Em. \pm & 0.37 & 0.34 & 0.39 & 0.29 & 0.01 & 0.02 & $\mathrm{R}_{1} \mathrm{~T}_{3}$ & 43.67 & 43.2 & 12.9 & 14.3 & 1.35 & 1.33 \\
\hline $\mathrm{CD}$ at $5 \%$ & 1.1 & 1.01 & 1.15 & 0.85 & 0.09 & 0.09 & $\mathrm{R}_{1} \mathrm{~T}_{4}$ & 46.47 & 46.96 & 16.5 & 18 & 1.29 & 1.32 \\
\hline \multicolumn{7}{|c|}{ Rotation system (R) } & $\mathrm{R}_{1} \mathrm{~T}_{5}$ & 42.2 & 42.49 & 16.5 & 16.2 & 1.41 & 1.42 \\
\hline $\mathrm{R}_{1}$ & 43.68 & 43.66 & 16.24 & 16.27 & 1.36 & 1.37 & $\mathrm{R}_{2} \mathrm{~T}_{1}$ & 40.27 & 41.47 & 15.18 & 18.5 & 1.39 & 1.4 \\
\hline $\mathrm{R}_{2}$ & 42.16 & 42.46 & 15.32 & 17.08 & 1.4 & 1.4 & $\mathrm{R}_{2} \mathrm{~T}_{2}$ & 42.22 & 42.41 & 14.3 & 16.7 & 1.42 & 1.42 \\
\hline S.Em. \pm & 0.37 & 0.34 & 0.39 & 0.29 & 0.01 & 0.02 & $\mathrm{R}_{2} \mathrm{~T}_{3}$ & 42.61 & 42.47 & 18.3 & 18.7 & 1.38 & 1.38 \\
\hline $\mathrm{CD}$ at $5 \%$ & 1.1 & 1.01 & NS & NS & NS & NS & $\mathrm{R}_{2} \mathrm{~T}_{4}$ & 44.44 & 44.75 & 12.9 & 14.8 & 1.37 & 1.37 \\
\hline \multicolumn{7}{|c|}{ Treatments (T) } & $\mathrm{R}_{2} \mathrm{~T}_{5}$ & 41.26 & 41.19 & 15.93 & 16.7 & 1.41 & 1.41 \\
\hline $\mathrm{T}_{1}$ & 41.73 & 41.76 & 16.18 & 17.55 & 1.39 & 1.4 & S.Em. \pm & 0.83 & 0.76 & 0.87 & 0.64 & 0.03 & 0.03 \\
\hline $\mathrm{T}_{2}$ & 42.56 & 43 & 16.2 & 16.48 & 1.4 & 1.39 & $\mathrm{CD}$ at $5 \%$ & NS & NS & 2.58 & 1.89 & NS & NS \\
\hline $\mathrm{T}_{3}$ & 43.14 & 42.83 & 15.6 & 16.5 & 1.36 & 1.36 & \multicolumn{7}{|c|}{ Interaction (SxRxT) } \\
\hline $\mathrm{T}_{4}$ & 45.46 & 45.86 & 14.7 & 16.4 & 1.33 & 1.34 & $\mathrm{~S}_{1} \mathrm{R}_{1} \mathrm{~T}_{1}$ & 43.73 & 44.15 & 17.75 & 16.2 & 1.35 & 1.38 \\
\hline $\mathrm{T}_{5}$ & 41.73 & 41.84 & 16.21 & 16.45 & 1.41 & 1.41 & $\mathrm{~S}_{1} \mathrm{R}_{1} \mathrm{~T}_{2}$ & 44.34 & 43.96 & 19.4 & 20.52 & 1.28 & 1.28 \\
\hline S.Em. \pm & 0.59 & 0.54 & 0.62 & 0.45 & 0.02 & 0.02 & $\mathrm{~S}_{1} \mathrm{R}_{1} \mathrm{~T}_{3}$ & 45.07 & 45.09 & 16.2 & 16.6 & 1.26 & 1.26 \\
\hline $\mathrm{CD}$ at $5 \%$ & 1.74 & 1.59 & NS & NS & NS & NS & $\mathrm{S}_{1} \mathrm{R}_{1} \mathrm{~T}_{4}$ & 46.37 & 47.2 & 23.6 & 24.6 & 1.26 & 1.27 \\
\hline \multicolumn{7}{|c|}{ Interaction (SxR) } & $\mathrm{S}_{1} \mathrm{R}_{1} \mathrm{~T}_{5}$ & 42.21 & 42.66 & 16.8 & 16.6 & 1.4 & 1.41 \\
\hline $\mathrm{S}_{1} \mathrm{R}_{1}$ & 44.34 & 44.61 & 18.75 & 18.9 & 1.31 & 1.32 & $\mathrm{~S}_{1} \mathrm{R}_{2} \mathrm{~T}_{1}$ & 40.67 & 42.45 & 14.75 & 21 & 1.36 & 1.36 \\
\hline $\mathrm{S}_{1} \mathrm{R}_{2}$ & 42.62 & 43.09 & 16.31 & 18.92 & 1.37 & 1.37 & $\mathrm{~S}_{1} \mathrm{R}_{2} \mathrm{~T}_{2}$ & 43.32 & 43.26 & 18.6 & 22.8 & 1.36 & 1.36 \\
\hline $\mathrm{S}_{2} \mathrm{R}_{1}$ & 43.02 & 42.7 & 13.72 & 13.64 & 1.42 & 1.41 & $\mathrm{~S}_{1} \mathrm{R}_{2} \mathrm{~T}_{3}$ & 42.54 & 42.89 & 21.4 & 23.2 & 1.36 & 1.37 \\
\hline $\mathrm{S}_{2} \mathrm{R}_{2}$ & 41.71 & 41.83 & 14.33 & 15.24 & 1.42 & 1.42 & $\mathrm{~S}_{1} \mathrm{R}_{2} \mathrm{~T}_{4}$ & 44.84 & 45.05 & 11 & 13.8 & 1.38 & 1.37 \\
\hline S.Em. \pm & 0.53 & 0.48 & 0.55 & 0.4 & 0.02 & 0.02 & $\mathrm{~S}_{1} \mathrm{R}_{2} \mathrm{~T}_{5}$ & 41.71 & 41.79 & 15.8 & 13.8 & 1.4 & 1.4 \\
\hline CD at $5 \%$ & NS & NS & 1.63 & NS & NS & NS & $\mathrm{S}_{2} \mathrm{R}_{1} \mathrm{~T}_{1}$ & 42.64 & 39.95 & 16.6 & 17 & 1.44 & 1.43 \\
\hline \multicolumn{7}{|c|}{ Interaction (SxT) } & $\mathrm{S}_{2} \mathrm{R}_{1} \mathrm{~T}_{2}$ & 41.45 & 43.2 & 16.8 & 12 & 1.46 & 1.44 \\
\hline$\overline{\mathrm{S}_{1} \mathrm{~T}_{1}}$ & 42.2 & 43.3 & 16.25 & 18.6 & 1.35 & 1.37 & $\mathrm{~S}_{2} \mathrm{R}_{1} \mathrm{~T}_{3}$ & 42.26 & 41.31 & 9.6 & 12 & 1.44 & 1.4 \\
\hline $\mathrm{S}_{1} \mathrm{~T}_{2}$ & 43.83 & 43.61 & 19 & 21.66 & 1.32 & 1.32 & $\mathrm{~S}_{2} \mathrm{R}_{1} \mathrm{~T}_{4}$ & 46.58 & 46.73 & 9.4 & 11.4 & 1.32 & 1.37 \\
\hline $\mathrm{S}_{1} \mathrm{~T}_{3}$ & 43.81 & 43.99 & 18.8 & 19.9 & 1.31 & 1.31 & $\mathrm{~S}_{2} \mathrm{R}_{1} \mathrm{~T}_{5}$ & 42.19 & 42.31 & 16.2 & 15.8 & 1.42 & 1.42 \\
\hline $\mathrm{S}_{1} \mathrm{~T}_{4}$ & 45.6 & 46.13 & 17.3 & 19.2 & 1.32 & 1.32 & $\mathrm{~S}_{2} \mathrm{R}_{2} \mathrm{~T}_{1}$ & 39.87 & 40.49 & 15.6 & 16 & 1.43 & 1.43 \\
\hline $\mathrm{S}_{1} \mathrm{~T}_{5}$ & 41.96 & 42.23 & 16.3 & 15.2 & 1.4 & 1.41 & $\mathrm{~S}_{2} \mathrm{R}_{2} \mathrm{~T}_{2}$ & 41.13 & 41.57 & 10 & 10.6 & 1.49 & 1.49 \\
\hline $\mathrm{S}_{2} \mathrm{~T}_{1}$ & 41.26 & 40.22 & 16.1 & 16.5 & 1.43 & 1.43 & $\mathrm{~S}_{2} \mathrm{R}_{2} \mathrm{~T}_{3}$ & 42.69 & 42.04 & 15.2 & 14.2 & 1.39 & 1.4 \\
\hline $\mathrm{S}_{2} \mathrm{~T}_{2}$ & 41.29 & 42.39 & 13.4 & 11.3 & 1.48 & 1.47 & $\mathrm{~S}_{2} \mathrm{R}_{2} \mathrm{~T}_{4}$ & 44.05 & 44.46 & 14.8 & 15.8 & 1.37 & 1.36 \\
\hline $\mathrm{S}_{2} \mathrm{~T}_{3}$ & 42.47 & 41.68 & 12.4 & 13.1 & 1.41 & 1.4 & $\mathrm{~S}_{2} \mathrm{R}_{2} \mathrm{~T}_{5}$ & 40.8 & 40.58 & 16.05 & 19.6 & 1.42 & 1.43 \\
\hline $\mathrm{S}_{2} \mathrm{~T}_{4}$ & 45.31 & 45.59 & 12.1 & 13.6 & 1.35 & 1.37 & S.Em.. & 1.18 & 1.08 & 1.23 & 0.9 & 0.05 & 0.05 \\
\hline $\mathrm{S}_{2} \mathrm{~T}_{5}$ & 41.5 & 41.45 & 16.13 & 17.7 & 1.42 & 1.42 & $\mathrm{CD}$ at $5 \%$ & NS & NS & 3.65 & 2.68 & NS & NS \\
\hline S.Em.. \pm & 0.83 & 0.76 & 0.87 & 0.64 & 0.03 & 0.03 & \multirow{2}{*}{\multicolumn{7}{|c|}{ NS: Non-significant }} \\
\hline $\mathrm{CD}$ at $5 \%$ & NS & NS & 2.58 & 1.89 & NS & NS & & & & & & & \\
\hline
\end{tabular}

$\mathrm{S}_{1}$ : FYM, $\mathrm{S}_{2}$ : Maize residue, B.D : Bulk density, $\mathrm{R}_{1}$ : Finger millet - groundnut rotation, $\mathrm{R}_{2}$ : Finger millet mono cropping, I.R: Infiltration rate, $\mathrm{T}_{1}$ : Absolute control, $\mathrm{T}_{2}$ : Organic matter, $\mathrm{T}_{3}$ : Organic matter $+50 \% \mathrm{~N}_{2} \mathrm{P}_{2} \mathrm{O}_{5}$ and $\mathrm{K}_{2} \mathrm{O}, \mathrm{T}_{4}$ : Organic matter + $100 \% \mathrm{~N}, \mathrm{P}_{2} \mathrm{O}_{5}$ and $\mathrm{K}_{2} \mathrm{O}, \mathrm{T}_{5}: 100 \% \mathrm{~N}, \mathrm{P}_{2} \mathrm{O}_{5}$ and $\mathrm{K}_{2} \mathrm{O}$

$\left.\mathrm{ha}^{-1}\right\}$ second factor was rotation system $\left\{\mathrm{R}_{1}\right.$ :- legume rotation and $\mathrm{R}_{2}$ :-mono cropping $\}$ and third factor was nutrient source with five levels \{ Absolute control $\left(\mathrm{T}_{1}\right)$, only organic matter $\left(\mathrm{T}_{2}\right), 50 \%$ recommended dose of fertilizer + organic matter $\left(\mathrm{T}_{3}\right), 100 \%$ recommended dose of fertilizer + organic matter $\left(\mathrm{T}_{4}\right)$ and only recommended dose of fertilizer $\left.\left(\mathrm{T}_{5}\right)\right\}$.

Soil samples were air dried and sieved through $2 \mathrm{~mm}$ sieve \& analysed to estimate soil reaction $(\mathrm{pH})$, Electrical conductivity $(\mathrm{Ec})$, organic carbon $(\mathrm{OC} \%)$, available soil N, available $\mathrm{P}$, and available $\mathrm{K}$ by the standard procedures. Microbial activities like Dehydrogenase, Urease, Acid and Alkaline phosphatase activities are analysed by the standard procedures of Casida et al. (1964) and Eivazi and Tabatabai (1977). Physical parameters of soil like infiltration rate was measured by double ring infiltrometer developed by Parr and Bertrand and maximum water holding capacity and bulk density was measured by keen's cup method de- veloped by Jackson (1956).

The organic matter like FYM and maize residue were applied at the time of final ploughing. Urea, SSP and MOP were used as nutrient sources. 50 per cent nitrogen was top-dressed at tillering stage, and rest half of the nitrogen and full $\mathrm{P}$ and $\mathrm{K}$ were applied as basal dose. GPU-28 variety of finger millet was sown with $10 \mathrm{~kg}$ per ha at a spacing of $30 \mathrm{~cm} \times 10 \mathrm{~cm}$. The growth parameters were recorded at thirty days interval and yield parameters were recorded at 90 DAS and at harvest. The experimental data collected on various growth and yield components were subjected to Fisher's method of "Analysis of variance" (ANOVA) as outlined by Gomez and Gomez (1984) and data were compared with critical differences at a probability level of 05 per cent.

\section{RESULTS AND DISCUSSION}

Maximum water holding capacity, infiltration rate 
Pavankumar Goudar et al. / J. Appl. \& Nat. Sci. 9 (2): 680 - 687 (2017)

Table 2. Soil chemical properties after harvest of the crop as influenced by legume rotation, use of organic and inorganic sources of nutrients.

\begin{tabular}{|c|c|c|c|c|c|c|c|}
\hline Treatment & pH & $E C\left({\left.d S m^{-1}\right)}\right.$ & OC (\%) & Treatment & $\mathrm{pH}$ & $E C\left(d S^{-1}\right)$ & OC (\%) \\
\hline \multicolumn{4}{|c|}{ Source of organic manure (S) } & \multicolumn{4}{|c|}{ Interaction (RxT) } \\
\hline $\mathrm{S}_{1}$ & 5.67 & 0.03 & 0.42 & $\mathrm{R}_{1} \mathrm{~T}_{1}$ & 5.64 & 0.02 & 0.38 \\
\hline $\mathrm{S}_{2}$ & 5.64 & 0.02 & 0.43 & $\mathrm{R}_{1} \mathrm{~T}_{2}$ & 5.67 & 0.02 & 0.44 \\
\hline S.Em. \pm & 0.1 & 0.001 & 0.01 & $\mathrm{R}_{1} \mathrm{~T}_{3}$ & 5.75 & 0.04 & 0.46 \\
\hline $\mathrm{CD}$ at $5 \%$ & NS & 0.003 & NS & $\mathrm{R}_{1} \mathrm{~T}_{4}$ & 5.58 & 0.03 & 0.51 \\
\hline \multicolumn{4}{|c|}{ Rotation system (R) } & $\mathrm{R}_{1} \mathrm{~T}_{5}$ & 5.66 & 0.03 & 0.39 \\
\hline $\mathrm{R}_{1}$ & 5.66 & 0.03 & 0.44 & $\mathrm{R}_{2} \mathrm{~T}_{1}$ & 5.51 & 0.01 & 0.28 \\
\hline $\mathrm{R}_{2}$ & 5.65 & 0.02 & 0.41 & $\mathrm{R}_{2} \mathrm{~T}_{2}$ & 5.91 & 0.02 & 0.44 \\
\hline S.Em. \pm & 0.1 & 0.001 & 0.01 & $\mathrm{R}_{2} \mathrm{~T}_{3}$ & 5.66 & 0.03 & 0.43 \\
\hline $\mathrm{CD}$ at $5 \%$ & NS & 0.003 & NS & $\mathrm{R}_{2} \mathrm{~T}_{4}$ & 5.46 & 0.02 & 0.54 \\
\hline \multicolumn{4}{|c|}{ Nutrient source $(T)$} & $\mathrm{R}_{2} \mathrm{~T}_{5}$ & 5.71 & 0.02 & 0.38 \\
\hline $\mathrm{T}_{1}$ & 5.58 & 0.02 & 0.33 & S.Em. \pm & 0.21 & 0.002 & 0.02 \\
\hline $\mathrm{T}_{2}$ & 5.79 & 0.02 & 0.44 & $\mathrm{CD}$ at $5 \%$ & NS & NS & NS \\
\hline $\mathrm{T}_{3}$ & 5.7 & 0.03 & 0.44 & \multicolumn{4}{|c|}{ Interaction (SxRxT) } \\
\hline $\mathrm{T}_{4}$ & 5.52 & 0.02 & 0.53 & $\mathrm{~S}_{1} \mathrm{R}_{1} \mathrm{~T}_{1}$ & 5.35 & 0.02 & 0.36 \\
\hline $\mathrm{T}_{5}$ & 5.68 & 0.02 & 0.38 & $\mathrm{~S}_{1} \mathrm{R}_{1} \mathrm{~T}_{2}$ & 5.65 & 0.03 & 0.5 \\
\hline S.Em. \pm & 0.15 & 0.002 & 0.02 & $\mathrm{~S}_{1} \mathrm{R}_{1} \mathrm{~T}_{3}$ & 5.74 & 0.05 & 0.53 \\
\hline $\mathrm{CD}$ at $5 \%$ & NS & 0.005 & 0.05 & $\mathrm{~S}_{1} \mathrm{R}_{1} \mathrm{~T}_{4}$ & 5.86 & 0.03 & 0.53 \\
\hline \multicolumn{4}{|c|}{ Interaction (SxR) } & $\mathrm{S}_{1} \mathrm{R}_{1} \mathrm{~T}_{5}$ & 5.89 & 0.03 & 0.35 \\
\hline $\mathrm{S}_{1} \mathrm{R}_{1}$ & 5.76 & 0.03 & 0.45 & $\mathrm{~S}_{1} \mathrm{R}_{2} \mathrm{~T}_{1}$ & 5.44 & 0.02 & 0.24 \\
\hline$S_{1} R_{2}$ & 5.58 & 0.03 & 0.39 & $\mathrm{~S}_{1} \mathrm{R}_{2} \mathrm{~T}_{2}$ & 5.61 & 0.03 & 0.42 \\
\hline $\mathrm{S}_{2} \mathrm{R}_{1}$ & 5.56 & 0.02 & 0.42 & $\mathrm{~S}_{1} \mathrm{R}_{2} \mathrm{~T}_{3}$ & 5.59 & 0.04 & 0.42 \\
\hline $\mathrm{S}_{2} \mathrm{R}_{2}$ & 5.72 & 0.02 & 0.43 & $\mathrm{~S}_{1} \mathrm{R}_{2} \mathrm{~T}_{4}$ & 5.54 & 0.03 & 0.57 \\
\hline S.Em. \pm & 0.14 & 0.001 & 0.01 & $\mathrm{~S}_{1} \mathrm{R}_{2} \mathrm{~T}_{5}$ & 5.71 & 0.02 & 0.32 \\
\hline $\mathrm{CD}$ at $5 \%$ & NS & NS & 0.04 & $\mathrm{~S}_{2} \mathrm{R}_{1} \mathrm{~T}_{1}$ & 5.3 & 0.02 & 0.39 \\
\hline \multicolumn{4}{|c|}{ Interaction (SxT) } & $\mathrm{S}_{2} \mathrm{R}_{1} \mathrm{~T}_{2}$ & 5.69 & 0.02 & 0.39 \\
\hline $\mathrm{S}_{1} \mathrm{~T}_{1}$ & 5.63 & 0.02 & 0.3 & $\mathrm{~S}_{2} \mathrm{R}_{1} \mathrm{~T}_{3}$ & 5.75 & 0.02 & 0.39 \\
\hline $\mathrm{S}_{1} \mathrm{~T}_{2}$ & 5.55 & 0.03 & 0.46 & $\mathrm{~S}_{2} \mathrm{R}_{1} \mathrm{~T}_{4}$ & 5.63 & 0.03 & 0.5 \\
\hline $\mathrm{S}_{1} \mathrm{~T}_{3}$ & 5.66 & 0.04 & 0.47 & $\mathrm{~S}_{2} \mathrm{R}_{1} \mathrm{~T}_{5}$ & 5.44 & 0.03 & 0.44 \\
\hline $\mathrm{S}_{1} \mathrm{~T}_{4}$ & 5.7 & 0.03 & 0.55 & $\mathrm{~S}_{2} \mathrm{R}_{2} \mathrm{~T}_{1}$ & 5.37 & 0.01 & 0.32 \\
\hline $\mathrm{S}_{1} \mathrm{~T}_{5}$ & 5.8 & 0.02 & 0.33 & $\mathrm{~S}_{2} \mathrm{R}_{2} \mathrm{~T}_{2}$ & 5.42 & 0.01 & 0.47 \\
\hline $\mathrm{S}_{2} \mathrm{~T}_{1}$ & 5.52 & 0.02 & 0.35 & $\mathrm{~S}_{2} \mathrm{R}_{2} \mathrm{~T}_{3}$ & 5.74 & 0.02 & 0.44 \\
\hline $\mathrm{S}_{2} \mathrm{~T}_{2}$ & 6.03 & 0.01 & 0.43 & $\mathrm{~S}_{2} \mathrm{R}_{2} \mathrm{~T}_{4}$ & 5.89 & 0.02 & 0.51 \\
\hline $\mathrm{S}_{2} \mathrm{~T}_{3}$ & 5.74 & 0.02 & 0.41 & $\mathrm{~S}_{2} \mathrm{R}_{2} \mathrm{~T}_{5}$ & 5.7 & 0.02 & 0.44 \\
\hline $\mathrm{S}_{2} \mathrm{~T}_{4}$ & 5.34 & 0.02 & 0.5 & S.Em. \pm & 0.3 & 0.003 & 0.03 \\
\hline $\mathrm{S}_{2} \mathrm{~T}_{5}$ & 5.57 & 0.02 & 0.44 & $\mathrm{CD}$ at $5 \%$ & NS & NS & NS \\
\hline S.Em. \pm & 0.21 & 0.002 & 0.02 & \multirow{2}{*}{\multicolumn{4}{|c|}{ NS: Non-significant }} \\
\hline $\mathrm{CD}$ at $5 \%$ & NS & 0.01 & 0.07 & & & & \\
\hline
\end{tabular}

$\mathrm{S}_{1}$ : FYM, $\mathrm{S}_{2}$ : Maize residue, $\mathrm{R}_{1}$ : Finger millet - groundnut rotation, $\mathrm{R}_{2}$ : Finger millet mono cropping, $\mathrm{T}_{1}$ : Absolute control, $\mathrm{T}_{2}$ : Organic matter, $\mathrm{T}_{3}$ : Organic matter $+50 \% \mathrm{~N}, \mathrm{P}_{2} \mathrm{O}_{5}$ and $\mathrm{K}_{2} \mathrm{O}, \mathrm{T}_{4}$ : Organic matter $+100 \% \mathrm{~N}, \mathrm{P}_{2} \mathrm{O}_{5}$ and $\mathrm{K}_{2} \mathrm{O}, \mathrm{T}_{5}: 100 \% \mathrm{~N}, \mathrm{P}_{2} \mathrm{O}_{5}$ and $\mathrm{K}_{2} \mathrm{O}$

and bulk density: 10 tonnes of FYM recorded significantly higher values of maximum water holding capacity and infiltration rate in both situations like before sowing and after harvest of the crop (43.48 and 43.85 $\%$ and $17.93,18.91 \mathrm{~cm} \mathrm{hr}^{-1}$ respectively) than the maize residue@ 5 tonnes ha ${ }^{-1}$ (42.37 and $42.27 \%$ and 14.03 and $14.44 \mathrm{~cm} \mathrm{hr}^{-1}$ respectively) at 5 per cent significance level. Application of fly ash 10 tonnes ha $^{-1}$ and 15 tonnes of FYM ha ${ }^{-1}$ decreased the bulk density significantly and increased the soil porosity, infiltration rate and water holding capacity of Vertisols of Coimbatore, (Birajdar et al., 2001). Further, the bulk density of soil decreased significantly with the application of farm yard manure or Sesbania aculeate either alone or in combination with inorganic fertilizers (Bajpai et al., 2006). Rotation system shows signif- icant difference in before sowing and after harvest of the crop with mono-cropped finger millet the maximum water holding capacity was significantly lower $(42.16 \%, 42.46 \%)$ than under rotation with legume crop $(43.68 \%, 43.66 \%)$ respectively at probability level of 0.05. Patidar and Mali (2014) reported that application of organic amendments such as FYM, poultry manure, maize straw and cotton waste significantly reduced the bulk density of soil. But the rotation system and nutrient sources failed to show the significant difference in infiltration rate. Maximum water holding capacity was significantly higher in $100 \%$ recommended dose of fertilizer + organic matter before sowing and after harvest of the crop (45.46\% and $45.86 \%$ respectively), than the absolute control ( 41.73 $\%$ and $41.76 \%$ ) and this was on par with the $100 \%$ 
Pavankumar Goudar et al. / J. Appl. \& Nat. Sci. 9 (2): 680 - 687 (2017)

Table 3. Dehydrogenase and Urease activity of soil as influenced by legume rotation, use of organic and inorganic sources of nutrients.

\begin{tabular}{|c|c|c|c|c|c|c|c|c|c|}
\hline & \multicolumn{2}{|c|}{ D. A $\left(\mu g\right.$ TPF g $\left.g^{-1} 24 h^{-1}\right)$} & \multicolumn{2}{|c|}{ U.A(g NH4 g ${ }^{-1}$ soil $)$} & & \multicolumn{2}{|c|}{ D. A $\left(\mu \mathrm{g}\right.$ TPF $\left.g^{-1} 24 \mathrm{~h}^{-1}\right)$} & \multicolumn{2}{|c|}{ U.A(g NH4 g $^{-1}$ soil $)$} \\
\hline & Before & After & Before & After & & Before & After & Before & After \\
\hline \multicolumn{5}{|c|}{ Source of organic manure $(\mathrm{S})$} & \multicolumn{5}{|c|}{ Interaction (RxT) } \\
\hline $\mathrm{S}_{1}$ & 374.13 & 357.60 & 24.59 & 23.90 & $\mathrm{R}_{1} \mathrm{~T}_{1}$ & 161.56 & 152.86 & 15.08 & 12.76 \\
\hline $\mathrm{S}_{2}$ & 203.54 & 193.00 & 20.53 & 18.10 & $\mathrm{R}_{1} \mathrm{~T}_{2}$ & 308.70 & 294.14 & 19.72 & 17.98 \\
\hline S.Em. \pm & 0.61 & 0.18 & 0.67 & 0.59 & $\mathrm{R}_{1} \mathrm{~T}_{3}$ & 391.22 & 379.63 & 23.78 & 22.62 \\
\hline $\mathrm{CD}$ at $5 \%$ & 1.80 & 0.53 & 1.99 & 1.75 & $\mathrm{R}_{1} \mathrm{~T}_{4}$ & 453.52 & 442.66 & 49.30 & 48.14 \\
\hline \multicolumn{5}{|c|}{ Rotation system $(\mathbf{R})$} & $\mathrm{R}_{1} \mathrm{~T}_{5}$ & 240.53 & 223.14 & 16.82 & 16.82 \\
\hline $\mathrm{R}_{1}$ & 311.11 & 298.48 & 24.94 & 23.66 & $\mathrm{R}_{2} \mathrm{~T}_{1}$ & 126.06 & 114.47 & 9.86 & 10.44 \\
\hline $\mathrm{R}_{2}$ & 266.57 & 252.12 & 20.18 & 18.33 & $\mathrm{R}_{2} \mathrm{~T}_{2}$ & 224.59 & 213.00 & 16.82 & 14.50 \\
\hline S.Em. \pm & 0.61 & 0.18 & 0.67 & 0.59 & $\mathrm{R}_{2} \mathrm{~T}_{3}$ & 361.51 & 341.23 & 18.56 & 16.24 \\
\hline CD at $5 \%$ & 1.80 & 0.53 & 1.99 & 1.75 & $\mathrm{R}_{2} \mathrm{~T}_{4}$ & 428.69 & 420.20 & 40.60 & 38.86 \\
\hline \multicolumn{5}{|c|}{ Treatments (T) } & $\mathrm{R}_{2} \mathrm{~T}_{5}$ & 191.99 & 171.70 & 15.08 & 11.60 \\
\hline$\overline{T_{1}}$ & 143.81 & 133.67 & 12.47 & 11.60 & S.Em. \pm & 1.36 & 0.40 & 1.50 & 1.32 \\
\hline $\mathrm{T}_{2}$ & 266.64 & 253.57 & 18.27 & 16.24 & $\mathrm{CD}$ at $5 \%$ & 4.02 & 1.19 & NS & NS \\
\hline $\mathrm{T}_{3}$ & 376.37 & 360.43 & 21.17 & 19.43 & \multicolumn{5}{|c|}{ Interaction (SxRxT) } \\
\hline $\mathrm{T}_{4}$ & 441.10 & 431.43 & 44.95 & 43.50 & $\mathrm{~S}_{1} \mathrm{R}_{1} \mathrm{~T}_{1}$ & 202.85 & 191.26 & 18.56 & 15.08 \\
\hline $\mathrm{T}_{5}$ & 216.26 & 197.42 & 15.95 & 14.21 & $\mathrm{~S}_{1} \mathrm{R}_{1} \mathrm{~T}_{2}$ & 369.64 & 362.24 & 19.72 & 19.72 \\
\hline S.Em. \pm & 0.96 & 0.29 & 1.06 & 0.93 & $\mathrm{~S}_{1} \mathrm{R}_{1} \mathrm{~T}_{3}$ & 539.01 & 520.17 & 24.36 & 25.52 \\
\hline CD at $5 \%$ & 2.85 & 0.84 & 3.14 & 2.76 & $\mathrm{~S}_{1} \mathrm{R}_{1} \mathrm{~T}_{4}$ & 628.85 & 611.46 & 53.36 & 55.68 \\
\hline \multicolumn{5}{|c|}{ Interaction (SxR) } & $\mathrm{S}_{1} \mathrm{R}_{1} \mathrm{~T}_{5}$ & 239.08 & 214.45 & 19.72 & 18.56 \\
\hline$\overline{S_{1} R_{1}}$ & 395.88 & 379.92 & 27.14 & 26.91 & $\mathrm{~S}_{1} \mathrm{R}_{2} \mathrm{~T}_{1}$ & 144.90 & 127.51 & 12.76 & 12.76 \\
\hline $\mathrm{S}_{1} \mathrm{R}_{2}$ & 352.38 & 335.29 & 22.04 & 20.88 & $\mathrm{~S}_{1} \mathrm{R}_{2} \mathrm{~T}_{2}$ & 305.73 & 295.59 & 17.40 & 16.24 \\
\hline $\mathrm{S}_{2} \mathrm{R}_{1}$ & 226.33 & 217.05 & 22.74 & 20.42 & $\mathrm{~S}_{1} \mathrm{R}_{2} \mathrm{~T}_{3}$ & 499.89 & 485.40 & 20.88 & 20.88 \\
\hline $\mathrm{S}_{2} \mathrm{R}_{2}$ & 180.75 & 168.95 & 18.33 & 15.78 & $\mathrm{~S}_{1} \mathrm{R}_{2} \mathrm{~T}_{4}$ & 594.07 & 579.58 & 46.40 & 42.92 \\
\hline S.Em. \pm & 0.86 & 0.26 & 0.95 & 0.83 & $\mathrm{~S}_{1} \mathrm{R}_{2} \mathrm{~T}_{5}$ & 217.34 & 188.36 & 12.76 & 11.60 \\
\hline $\mathrm{CD}$ at $5 \%$ & NS & 0.76 & NS & NS & $\mathrm{S}_{2} \mathrm{R}_{1} \mathrm{~T}_{1}$ & 120.26 & 114.47 & 11.60 & 10.44 \\
\hline \multicolumn{5}{|c|}{ Interaction (SxT) } & $\mathrm{S}_{2} \mathrm{R}_{1} \mathrm{~T}_{2}$ & 247.77 & 226.04 & 19.72 & 16.24 \\
\hline $\mathrm{S}_{1} \mathrm{~T}_{1}$ & 173.87 & 159.39 & 15.66 & 13.92 & $\mathrm{~S}_{2} \mathrm{R}_{1} \mathrm{~T}_{3}$ & 243.42 & 239.08 & 23.20 & 19.72 \\
\hline $\mathrm{S}_{1} \mathrm{~T}_{2}$ & 337.68 & 328.91 & 18.56 & 17.98 & $\mathrm{~S}_{2} \mathrm{R}_{1} \mathrm{~T}_{4}$ & 278.20 & 273.85 & 45.24 & 40.60 \\
\hline $\mathrm{S}_{1} \mathrm{~T}_{3}$ & 519.45 & 502.79 & 22.62 & 23.20 & $\mathrm{~S}_{2} \mathrm{R}_{1} \mathrm{~T}_{5}$ & 241.98 & 231.83 & 13.92 & 15.08 \\
\hline $\mathrm{S}_{1} \mathrm{~T}_{4}$ & 611.46 & 595.52 & 49.88 & 49.30 & $\mathrm{~S}_{2} \mathrm{R}_{2} \mathrm{~T}_{1}$ & 107.22 & 101.43 & 6.96 & 8.12 \\
\hline $\mathrm{S}_{1} \mathrm{~T}_{5}$ & 228.21 & 201.40 & 16.24 & 15.08 & $\mathrm{~S}_{2} \mathrm{R}_{2} \mathrm{~T}_{2}$ & 143.45 & 130.41 & 16.24 & 12.76 \\
\hline $\mathrm{S}_{2} \mathrm{~T}_{1}$ & 113.74 & 107.95 & 9.28 & 9.28 & $\mathrm{~S}_{2} \mathrm{R}_{2} \mathrm{~T}_{3}$ & 223.14 & 197.06 & 16.24 & 11.60 \\
\hline $\mathrm{S}_{2} \mathrm{~T}_{2}$ & 195.61 & 178.22 & 17.98 & 14.50 & $\mathrm{~S}_{2} \mathrm{R}_{2} \mathrm{~T}_{4}$ & 263.31 & 260.81 & 34.80 & 34.80 \\
\hline $\mathrm{S}_{2} \mathrm{~T}_{3}$ & 233.28 & 218.07 & 19.72 & 15.66 & $\mathrm{~S}_{2} \mathrm{R}_{2} \mathrm{~T}_{5}$ & 166.63 & 155.04 & 17.40 & 11.60 \\
\hline $\mathrm{S}_{2} \mathrm{~T}_{4}$ & 270.75 & 267.33 & 40.02 & 37.70 & S.Em. \pm & 1.92 & 0.57 & 2.12 & 1.87 \\
\hline $\mathrm{S}_{2} \mathrm{~T}_{5}$ & 204.30 & 193.44 & 15.66 & 13.34 & $\mathrm{CD}$ at $5 \%$ & 5.69 & 1.69 & NS & NS \\
\hline S.Em. \pm & 1.36 & 0.40 & 1.50 & 1.32 & & & & & \\
\hline $\mathrm{CD}$ at $5 \%$ & 4.02 & 1.19 & 4.45 & 3.91 & \multicolumn{5}{|c|}{ NS: Non-significant } \\
\hline
\end{tabular}

$\mathrm{S}_{1}$ : FYM, $\mathrm{S}_{2}$ : Maize residue, D. A : Dehydrogenase activity, $\mathrm{R}_{1}$ : Finger millet - groundnut rotation, $\mathrm{R}_{2}$ : Finger millet mono cropping, U.A : Urease activity, $\mathrm{T}_{1}$ : Absolute control, $\mathrm{T}_{2}$ : Organic matter, $\mathrm{T}_{3}$ : Organic matter $+50 \% \mathrm{~N}_{2} \mathrm{P}_{2} \mathrm{O}_{5}$ and $\mathrm{K}_{2} \mathrm{O}, \mathrm{T}_{4}$ : Organic matter $+100 \% \mathrm{~N}, \mathrm{P}_{2} \mathrm{O}_{5}$ and $\mathrm{K}_{2} \mathrm{O}, \mathrm{T}_{5}: 100 \% \mathrm{~N}, \mathrm{P}_{2} \mathrm{O}_{5}$ and $\mathrm{K}_{2} \mathrm{O}$

recommended dose of fertilizer $(41.73 \%$ and 41.84 $\%$ ), only organic matter (42.56\% and $43 \%$ ) and $50 \%$ recommended dose of fertilizer + organic matter (43.14\% and $42.85 \%$ ) at 5 per cent level of significance. All the interaction effects were found to be nonsignificant. But in infiltration rate except organic source $\mathrm{x}$ rotation system all other interactions were found to be significant. The highest infiltration rate was noticed in the FYM @ 10 tonnes ha ${ }^{-1}$ with 100\% recommended dose of fertilizer grown in the rotation system $\left(24.60 \mathrm{~cm} \mathrm{hr}^{-1}\right)$ than the maize residue with only organic matter in the mono-cropping (12.00 cm $\mathrm{hr}^{-1}$ ) at $0.05 \mathrm{P}$. The main reasons were the higher amount of the organic matter will improve the soil biota, which in turns help in the improvement in the soil physical parameters. Further, it was recognized that the addition of higher amount of organic source will improve the soil porosity and consequently the infiltration rate. There was no significant difference in bulk density due to different organic sources, rotation systems, nutrient sources and their interactions.

Organic carbon $(\%)$, electrical conductivity $\left(\mathbf{d S m}^{-1}\right)$ and $\mathbf{p H}$ : Organic carbon after harvest of the crop was significantly $(0.05 \mathrm{P})$ higher in $100 \%$ recommended dose of fertilizer + organic matter $(0.53 \%)$ and the least was observed in the absolute control $(0.33$ $\%$ ) which was on par with only recommended dose of fertilizer alone $(0.38 \%)$. Organic carbon in soil after harvest of crop did not find any significant difference in the rotation system and organic source. However, the numerically higher value was observed in the legume rotation, 10 tonnes of FYM incorporation $(0.42$ and $0.44 \%$ ) than the mono-cropped, and with 5 tonnes of FYM ha ${ }^{-1}(0.41$ and $0.41 \%)$. All the interaction 
Pavankumar Goudar et al. / J. Appl. \& Nat. Sci. 9 (2): 680 - 687 (2017)

Table 4. Acid and Alkaline phosphatase activity of soil as influenced by legume rotation, use of organic and inorganic sources of nutrients.

\begin{tabular}{|c|c|c|c|c|c|c|c|c|c|}
\hline \multirow{2}{*}{ Treatment } & \multicolumn{2}{|c|}{ A.A (g PNP g ${ }^{-1}$ soil) } & \multicolumn{2}{|c|}{ A.P.A (g PNP g ${ }^{-1}$ soil) } & \multirow{2}{*}{ Treatment } & \multicolumn{2}{|c|}{ A.A (g PNP g ${ }^{-1}$ soil) } & \multicolumn{2}{|c|}{ A.P.A (g PNP g g ${ }^{-1}$ soil) } \\
\hline & Before & After & Before & After & & Before & After & Before & After \\
\hline \multicolumn{5}{|c|}{ Source of organic manure (S) } & \multicolumn{5}{|c|}{ Interaction (RxT) } \\
\hline $\mathrm{S}_{1}$ & 32.60 & 29.96 & 25.60 & 24.30 & $\mathrm{R}_{1} \mathrm{~T}_{1}$ & 25.30 & 23.42 & 16.59 & 15.77 \\
\hline $\mathrm{S}_{2}$ & 19.30 & 18.51 & 15.43 & 14.93 & $\mathrm{R}_{1} \mathrm{~T}_{2}$ & 31.38 & 32.88 & 22.82 & 21.63 \\
\hline S.Em. \pm & 0.62 & 0.32 & 0.32 & 0.27 & $\mathrm{R}_{1} \mathrm{~T}_{3}$ & 36.01 & 32.39 & 25.54 & 24.90 \\
\hline $\mathrm{CD}$ at $5 \%$ & 1.82 & 0.96 & 0.95 & 0.78 & $\mathrm{R}_{1} \mathrm{~T}_{4}$ & 37.61 & 34.58 & 40.73 & 37.16 \\
\hline \multicolumn{5}{|c|}{ Rotation system (R) } & $\mathrm{R}_{1} \mathrm{~T}_{5}$ & 17.55 & 17.55 & 18.00 & 17.23 \\
\hline $\mathrm{R}_{1}$ & 29.57 & 28.16 & 24.74 & 23.34 & $\mathrm{R}_{2} \mathrm{~T}_{1}$ & 18.43 & 16.57 & 12.03 & 11.85 \\
\hline $\mathrm{R}_{2}$ & 22.34 & 20.31 & 16.30 & 15.89 & $\mathrm{R}_{2} \mathrm{~T}_{2}$ & 25.47 & 23.88 & 16.19 & 16.05 \\
\hline S.Em. \pm & 0.62 & 0.32 & 0.32 & 0.27 & $\mathrm{R}_{2} \mathrm{~T}_{3}$ & 26.92 & 24.44 & 17.04 & 16.63 \\
\hline $\mathrm{CD}$ at $5 \%$ & 1.82 & 0.96 & 0.95 & 0.78 & $\mathrm{R}_{2} \mathrm{~T}_{4}$ & 25.47 & 23.18 & 24.86 & 24.20 \\
\hline \multicolumn{5}{|c|}{ Treatments (T) } & $\mathrm{R}_{2} \mathrm{~T}_{5}$ & 15.38 & 13.47 & 11.36 & 10.72 \\
\hline $\mathrm{T}_{1}$ & 21.86 & 20.00 & 14.31 & 13.81 & S.Em. \pm & 1.38 & 0.72 & 0.72 & 0.59 \\
\hline $\mathrm{T}_{2}$ & 28.43 & 28.38 & 19.51 & 18.84 & $\mathrm{CD}$ at $5 \%$ & 4.07 & 2.14 & 2.12 & 1.75 \\
\hline $\mathrm{T}_{3}$ & 31.47 & 28.41 & 21.29 & 20.77 & \multicolumn{5}{|c|}{ Interaction (SxRxT) } \\
\hline $\mathrm{T}_{4}$ & 31.54 & 28.88 & 32.79 & 30.68 & $\mathrm{~S}_{1} \mathrm{R}_{1} \mathrm{~T}_{1}$ & 32.63 & 30.74 & 18.73 & 18.09 \\
\hline $\mathrm{T}_{5}$ & 16.47 & 15.51 & 14.68 & 13.97 & $\mathrm{~S}_{1} \mathrm{R}_{1} \mathrm{~T}_{2}$ & 37.35 & 41.08 & 28.20 & 26.96 \\
\hline S.Em. \pm & 0.97 & 0.51 & 0.51 & 0.42 & $\mathrm{~S}_{1} \mathrm{R}_{1} \mathrm{~T}_{3}$ & 46.43 & 42.08 & 35.72 & 34.64 \\
\hline CD at $5 \%$ & 2.88 & 1.51 & 1.50 & 1.24 & $\mathrm{~S}_{1} \mathrm{R}_{1} \mathrm{~T}_{4}$ & 50.67 & 47.41 & 55.10 & 48.89 \\
\hline \multicolumn{5}{|c|}{ Interaction (SxR) } & $\mathrm{S}_{1} \mathrm{R}_{1} \mathrm{~T}_{5}$ & 22.08 & 18.01 & 21.53 & 20.13 \\
\hline $\mathrm{S}_{1} \mathrm{R}_{1}$ & 37.83 & 35.86 & 31.86 & 29.74 & $\mathrm{~S}_{1} \mathrm{R}_{2} \mathrm{~T}_{1}$ & 22.63 & 19.08 & 12.31 & 12.11 \\
\hline $\mathrm{S}_{1} \mathrm{R}_{2}$ & 27.37 & 24.06 & 19.34 & 18.86 & $\mathrm{~S}_{1} \mathrm{R}_{2} \mathrm{~T}_{2}$ & 32.63 & 30.51 & 16.37 & 16.26 \\
\hline $\mathrm{S}_{2} \mathrm{R}_{1}$ & 21.31 & 20.46 & 17.62 & 16.94 & $\mathrm{~S}_{1} \mathrm{R}_{2} \mathrm{~T}_{3}$ & 33.74 & 30.64 & 21.78 & 21.52 \\
\hline $\mathrm{S}_{2} \mathrm{R}_{2}$ & 17.30 & 16.56 & 13.25 & 12.92 & $\mathrm{~S}_{1} \mathrm{R}_{2} \mathrm{~T}_{4}$ & 29.07 & 25.89 & 32.94 & 32.00 \\
\hline S.Em. \pm & 0.87 & 0.46 & 0.45 & 0.37 & $\mathrm{~S}_{1} \mathrm{R}_{2} \mathrm{~T}_{5}$ & 18.81 & 14.18 & 13.31 & 12.40 \\
\hline $\mathrm{CD}$ at $5 \%$ & 2.57 & 1.35 & 1.34 & 1.11 & $\mathrm{~S}_{2} \mathrm{R}_{1} \mathrm{~T}_{1}$ & 17.96 & 16.10 & 14.45 & 13.45 \\
\hline \multicolumn{5}{|c|}{ Interaction (SxT) } & $\mathrm{S}_{2} \mathrm{R}_{1} \mathrm{~T}_{2}$ & 25.41 & 24.67 & 17.44 & 16.31 \\
\hline $\mathrm{S}_{1} \mathrm{~T}_{1}$ & 27.63 & 24.91 & 15.52 & 15.10 & $\mathrm{~S}_{2} \mathrm{R}_{1} \mathrm{~T}_{3}$ & 25.60 & 22.69 & 15.37 & 15.17 \\
\hline $\mathrm{S}_{1} \mathrm{~T}_{2}$ & 34.99 & 35.80 & 22.28 & 21.61 & $\mathrm{~S}_{2} \mathrm{R}_{1} \mathrm{~T}_{4}$ & 24.55 & 21.74 & 26.36 & 25.44 \\
\hline $\mathrm{S}_{1} \mathrm{~T}_{3}$ & 40.08 & 36.36 & 28.75 & 28.08 & $\mathrm{~S}_{2} \mathrm{R}_{1} \mathrm{~T}_{5}$ & 13.03 & 17.08 & 14.48 & 14.32 \\
\hline $\mathrm{S}_{1} \mathrm{~T}_{4}$ & 39.87 & 36.65 & 44.02 & 40.44 & $\mathrm{~S}_{2} \mathrm{R}_{2} \mathrm{~T}_{1}$ & 14.23 & 14.06 & 11.76 & 11.59 \\
\hline $\mathrm{S}_{1} \mathrm{~T}_{5}$ & 20.44 & 16.09 & 17.42 & 16.27 & $\mathrm{~S}_{2} \mathrm{R}_{2} \mathrm{~T}_{2}$ & 18.31 & 17.24 & 16.01 & 15.85 \\
\hline $\mathrm{S}_{2} \mathrm{~T}_{1}$ & 16.09 & 15.08 & 13.10 & 12.52 & $\mathrm{~S}_{2} \mathrm{R}_{2} \mathrm{~T}_{3}$ & 20.11 & 18.25 & 12.29 & 11.74 \\
\hline $\mathrm{S}_{2} \mathrm{~T}_{2}$ & 21.86 & 20.96 & 16.73 & 16.08 & $\mathrm{~S}_{2} \mathrm{R}_{2} \mathrm{~T}_{4}$ & 21.88 & 20.48 & 16.78 & 16.40 \\
\hline $\mathrm{S}_{2} \mathrm{~T}_{3}$ & 22.85 & 20.47 & 13.83 & 13.46 & $\mathrm{~S}_{2} \mathrm{R}_{2} \mathrm{~T}_{5}$ & 11.95 & 12.77 & 9.41 & 9.04 \\
\hline $\mathrm{S}_{2} \mathrm{~T}_{4}$ & 23.21 & 21.11 & 21.57 & 20.92 & S.Em. \pm & 1.94 & 1.02 & 1.01 & 0.84 \\
\hline $\mathrm{S}_{2} \mathrm{~T}_{5}$ & 12.49 & 14.93 & 11.94 & 11.68 & $\mathrm{CD}$ at $5 \%$ & 5.76 & 3.03 & 3.00 & 2.48 \\
\hline S.Em. \pm & 1.38 & 0.72 & 0.72 & 0.59 & \multirow{2}{*}{\multicolumn{5}{|c|}{ NS: Non-significant }} \\
\hline $\mathrm{CD}$ at $5 \%$ & 4.07 & 2.14 & 2.12 & 1.75 & & & & & \\
\hline
\end{tabular}

$\mathrm{S}_{1}$ : FYM, $\mathrm{S}_{2}$ : Maize residue, A. A : Acid phosphatase activity, $\mathrm{R}_{1}$ : Finger millet - groundnut rotation, $\mathrm{R}_{2}$ : Finger millet mono cropping, A.P.A: Alkaline phosphatase activity, $\mathrm{T}_{1}$ : Absolute control, $\mathrm{T}_{2}$ : Organic matter, $\mathrm{T}_{3}$ : Organic matter $+50 \% \mathrm{~N}, \mathrm{P}_{2} \mathrm{O}_{5}$ and $\mathrm{K}_{2} \mathrm{O}, \mathrm{T}_{4}$ : Organic matter $+100 \% \mathrm{~N}, \mathrm{P}_{2} \mathrm{O}_{5}$ and $\mathrm{K}_{2} \mathrm{O}, \mathrm{T}_{5}: 100 \% \mathrm{~N}, \mathrm{P}_{2} \mathrm{O}_{5}$ and $\mathrm{K}_{2} \mathrm{O}$

effects were found to be non-significant. The main reason for higher uptake was soil organic carbon and $\mathrm{pH}$. The higher soil organic carbon is because of the bulky nature of the FYM. Organic carbon will improve the soil structure and physical parameters of the soil. Finger millet $(0.48 \%)$ than the mono cropped finger millet. The main reason behind these was the organic matter incorporated by the legume crop to the soil in the form of leaves and the roots. Hence, the availability of nutrients has higher in legume rotation. Dhull et al. (2005) after taking three successive crops found that soil organic $\mathrm{C}$ and total $\mathrm{N}$ level increased in the rotation than taking two crops. Microbial biomass $\mathrm{C}$ and dehydrogenase activity were higher in pearl millet - wheat-green manure (legume) rotation and was lower in pearl millet- wheat rotation. Electrical conductivity in soil did not show significant difference in the organ- ic and inorganic nutrient sources and the legume rotation and their interactions. However, the $\mathrm{pH}$ ranged from the 5.32 to 5.70 and there is no significance relation in between. The good soil condition and the nutrient availability of the legume rotated crop significantly increased with the microbial activities like dehydrogenase activity etc.

Dehydrogenase activity ( $\mu$ g TPF $\mathrm{g}^{-1} 24 \mathrm{~h}^{-1}$ ) and urease activity (g $\mathrm{NH}_{4} \mathbf{g}^{-1}$ soil): Incorporation of 10 tonnes of FYM recorded significantly higher dehydrogenase activity and urease activity in soil before sowing and after harvest of the crop $(374.13,357.60 \mu \mathrm{g}$ TPF g $\mathrm{g}^{-1} 24 \mathrm{~h}^{-1}$ and $24.59,23.90 \mathrm{~g} \mathrm{NH}_{4} \mathrm{~g}^{-1}$ respectively) than maize residue @ 5 tonnes ha ${ }^{-1}(203.54193 .01 \mu \mathrm{g}$ TPF $\mathrm{g}^{-1} 24 \mathrm{~h}^{-1}$ and $20.53,18.10 \mathrm{~g} \mathrm{NH}_{4} \mathrm{~g}^{-1}$ respectively) at 5 percent probability. Legume rotation system in finger millet has shown significantly higher $(0.05 \mathrm{P})$ 
Pavankumar Goudar et al. / J. Appl. \& Nat. Sci. 9 (2): 680 - 687 (2017)

Table 5. Ear head length, grain weight hill $^{-1}$, Grain yield and Straw yield of finger millet as influenced by legume rotation, use of organic and inorganic sources of nutrients.

\begin{tabular}{|c|c|c|c|c|c|c|c|c|c|}
\hline Treatment & $\begin{array}{l}\text { Ear head } \\
\text { length } \\
(\mathrm{cm})\end{array}$ & $\begin{array}{l}\text { Grain wt } \\
\text { hill }^{-1}(g)\end{array}$ & $\begin{array}{l}\text { Grain } \\
\text { yield } \\
\text { (kg ha } \\
\text { 1) }\end{array}$ & $\begin{array}{l}\text { Straw } \\
\text { yield } \\
(\mathrm{kg} \mathrm{ha} \\
\text { 1) }\end{array}$ & Treatment & $\begin{array}{l}\text { Ear } \\
\text { head } \\
\text { length } \\
(\mathrm{cm}) \\
\end{array}$ & $\begin{array}{l}\text { Grain } \\
\text { wt hill } \\
\text { (g) }\end{array}$ & $\begin{array}{l}\text { Grain } \\
\text { yield } \\
\left(\mathrm{kg} \mathrm{ha}^{-1}\right)\end{array}$ & $\begin{array}{l}\text { Straw } \\
\text { yield } \\
\left(\mathrm{kg} \mathrm{ha}^{-}\right. \\
\left.\mathbf{1}^{1}\right)\end{array}$ \\
\hline \multicolumn{5}{|c|}{ Source of organic manure (S) } & \multicolumn{5}{|c|}{ Interaction (RxT) } \\
\hline $\mathrm{S}_{1}$ & 7.25 & 9.10 & 1761 & 2672 & $\mathrm{R}_{1} \mathrm{~T}_{1}$ & 6.57 & 6.03 & 238 & 309 \\
\hline $\mathrm{S}_{2}$ & 6.66 & 7.16 & 1365 & 2218 & $\mathrm{R}_{1} \mathrm{~T}_{2}$ & 6.89 & 9.30 & 1130 & 1571 \\
\hline S.Em. \pm & 0.13 & 0.19 & 59 & 82 & $\mathrm{R}_{1} \mathrm{~T}_{3}$ & 7.52 & 8.83 & 2231 & 4093 \\
\hline $\mathrm{CD}$ at $5 \%$ & 0.39 & 0.55 & 175 & 242 & $\mathrm{R}_{1} \mathrm{~T}_{4}$ & 8.23 & 11.65 & 3100 & 5322 \\
\hline \multicolumn{5}{|c|}{ Rotation system (R) } & $\mathrm{R}_{1} \mathrm{~T}_{5}$ & 7.26 & 11.85 & 11.55 & 30.66 \\
\hline $\mathrm{R}_{1}$ & 7.30 & 9.38 & 1783 & 2900 & $\mathrm{R}_{2} \mathrm{~T}_{1}$ & 6.30 & 3.48 & 217 & 203 \\
\hline $\mathrm{R}_{2}$ & 6.62 & 6.87 & 1343 & 1990 & $\mathrm{R}_{2} \mathrm{~T}_{2}$ & 6.02 & 5.70 & 872 & 920 \\
\hline S.Em. \pm & 0.13 & 0.19 & 59 & 82 & $\mathrm{R}_{2} \mathrm{~T}_{3}$ & 7.03 & 5.93 & 1648 & 2397 \\
\hline $\mathrm{CD}$ at $5 \%$ & 0.39 & 0.55 & 175 & 242 & $\mathrm{R}_{2} \mathrm{~T}_{4}$ & 7.61 & 10.50 & 2382 & 3874 \\
\hline \multicolumn{5}{|c|}{ Nutrient source (T) } & $\mathrm{R}_{2} \mathrm{~T}_{5}$ & 5.76 & 10.86 & 9.55 & 26.16 \\
\hline $\mathrm{T}_{1}$ & 6.44 & 4.75 & 227 & 256 & S.Em. \pm & 0.29 & 0.42 & 132 & 183 \\
\hline $\mathrm{T}_{2}$ & 6.45 & 7.50 & 1001 & 1245 & $\mathrm{CD}$ at $5 \%$ & NS & NS & NS & 540 \\
\hline $\mathrm{T}_{3}$ & 7.27 & 7.38 & 1939 & 3245 & \multicolumn{5}{|c|}{ Interaction (SxRxT) } \\
\hline $\mathrm{T}_{4}$ & 7.92 & 11.08 & 2741 & 4598 & $\mathrm{~S}_{1} \mathrm{R}_{1} \mathrm{~T}_{1}$ & 6.84 & 6.55 & 320 & 391 \\
\hline $\mathrm{T}_{5}$ & 6.71 & 9.93 & 1905 & 2881 & $\mathrm{~S}_{1} \mathrm{R}_{1} \mathrm{~T}_{2}$ & 7.48 & 13.45 & 1796 & 2604 \\
\hline S.Em. \pm & 0.21 & 0.30 & 94 & 129 & $\mathrm{~S}_{1} \mathrm{R}_{1} \mathrm{~T}_{3}$ & 7.85 & 11.10 & 2378 & 4541 \\
\hline $\mathrm{CD}$ at $5 \%$ & 0.62 & 0.88 & 277 & 382 & $\mathrm{~S}_{1} \mathrm{R}_{1} \mathrm{~T}_{4}$ & 8.95 & 13.75 & 3244 & 5143 \\
\hline \multicolumn{5}{|c|}{ Interaction (SxR) } & $\mathrm{S}_{1} \mathrm{R}_{1} \mathrm{~T}_{5}$ & 7.30 & 12.75 & 13.30 & 33.35 \\
\hline $\mathrm{S}_{1} \mathrm{R}_{1}$ & 7.76 & 11.40 & 1959 & 3161 & $\mathrm{~S}_{1} \mathrm{R}_{2} \mathrm{~T}_{1}$ & 6.55 & 2.05 & 345 & 326 \\
\hline $\mathrm{S}_{1} \mathrm{R}_{2}$ & 6.74 & 6.79 & 1562 & 2183 & $\mathrm{~S}_{1} \mathrm{R}_{2} \mathrm{~T}_{2}$ & 6.20 & 5.75 & 1499 & 1514 \\
\hline $\mathrm{S}_{2} \mathrm{R}_{1}$ & 6.84 & 7.36 & 1607 & 2640 & $\mathrm{~S}_{1} \mathrm{R}_{2} \mathrm{~T}_{3}$ & 7.15 & 4.35 & 1814 & 2596 \\
\hline $\mathrm{S}_{2} \mathrm{R}_{2}$ & 6.49 & 6.95 & 1123 & 1797 & $\mathrm{~S}_{1} \mathrm{R}_{2} \mathrm{~T}_{4}$ & 7.25 & 12.90 & 2448 & 3776 \\
\hline S.Em. \pm & 0.19 & 0.26 & 84 & 115 & $\mathrm{~S}_{1} \mathrm{R}_{2} \mathrm{~T}_{5}$ & 6.55 & 8.55 & 1707 & 2702 \\
\hline $\mathrm{CD}$ at $5 \%$ & NS & 0.78 & NS & NS & $\mathrm{S}_{2} \mathrm{R}_{1} \mathrm{~T}_{1}$ & 6.30 & 5.50 & 155 & 228 \\
\hline \multicolumn{5}{|c|}{ Interaction (SxT) } & $\mathrm{S}_{2} \mathrm{R}_{1} \mathrm{~T}_{2}$ & 6.09 & 12.43 & 5.65 & 24.17 \\
\hline$\overline{\mathrm{S}_{1} \mathrm{~T}_{1}}$ & 6.70 & 4.30 & 333 & 358 & $\mathrm{~S}_{2} \mathrm{R}_{1} \mathrm{~T}_{3}$ & 7.18 & 9.80 & 2083 & 3646 \\
\hline $\mathrm{S}_{1} \mathrm{~T}_{2}$ & 6.84 & 9.35 & 1647 & 2059 & $\mathrm{~S}_{2} \mathrm{R}_{1} \mathrm{~T}_{4}$ & 7.50 & 6.55 & 2957 & 5501 \\
\hline $\mathrm{S}_{1} \mathrm{~T}_{3}$ & 7.50 & 7.73 & 2096 & 3569 & $\mathrm{~S}_{2} \mathrm{R}_{1} \mathrm{~T}_{5}$ & 6.90 & 9.80 & 2376 & 3288 \\
\hline $\mathrm{S}_{1} \mathrm{~T}_{4}$ & 8.10 & 13.38 & 2846 & 4460 & $\mathrm{~S}_{2} \mathrm{R}_{2} \mathrm{~T}_{1}$ & 6.05 & 4.90 & 89 & 81 \\
\hline $\mathrm{S}_{1} \mathrm{~T}_{5}$ & 7.11 & 10.73 & 1881 & 2913 & $\mathrm{~S}_{2} \mathrm{R}_{2} \mathrm{~T}_{2}$ & 5.84 & 10.30 & 245 & 326 \\
\hline $\mathrm{S}_{2} \mathrm{~T}_{1}$ & 6.18 & 5.20 & 122 & 155 & $\mathrm{~S}_{2} \mathrm{R}_{2} \mathrm{~T}_{3}$ & 6.90 & 10.30 & 1482 & 2197 \\
\hline $\mathrm{S}_{2} \mathrm{~T}_{2}$ & 6.07 & 5.65 & 355 & 431 & $\mathrm{~S}_{2} \mathrm{R}_{2} \mathrm{~T}_{4}$ & 7.97 & 12.95 & 2316 & 3971 \\
\hline $\mathrm{S}_{2} \mathrm{~T}_{3}$ & 7.04 & 7.03 & 1782 & 2921 & $\mathrm{~S}_{2} \mathrm{R}_{2} \mathrm{~T}_{5}$ & 5.70 & 10.55 & 1482 & 2409 \\
\hline $\mathrm{S}_{2} \mathrm{~T}_{4}$ & 7.73 & 8.78 & 2636 & 4736 & S.Em. \pm & 0.42 & 0.59 & 187 & 258 \\
\hline $\mathrm{S}_{2} \mathrm{~T}_{5}$ & 6.30 & 9.13 & 1929 & 2848 & $\mathrm{CD}$ at $5 \%$ & NS & 1.75 & NS & NS \\
\hline S.Em. \pm & 0.29 & 0.42 & 132 & 183 & \multirow{2}{*}{\multicolumn{5}{|c|}{ NS: Non-significant }} \\
\hline $\mathrm{CD}$ at $5 \%$ & NS & 1.24 & 392 & 540 & & & & & \\
\hline
\end{tabular}

$\mathrm{S}_{1}$ : FYM, $\mathrm{S}_{2}$ : Maize residue, $\mathrm{R}_{1}$ : Finger millet - groundnut rotation, $\mathrm{R}_{2}$ : Finger millet mono cropping, $\mathrm{T}_{1}:$ Absolute control, $\mathrm{T}_{2}$ : Organic matter, $\mathrm{T}_{3}$ : Organic matter $+50 \% \mathrm{~N}, \mathrm{P}_{2} \mathrm{O}_{5}$ and $\mathrm{K}_{2} \mathrm{O}, \mathrm{T}_{4}$ : Organic matter $+100 \% \mathrm{~N}, \mathrm{P}_{2} \mathrm{O}_{5}$ and $\mathrm{K}_{2} \mathrm{O}, \mathrm{T}_{5}: 100 \% \mathrm{~N}_{2} \mathrm{P}_{2} \mathrm{O}_{5}$ and $\mathrm{K}_{2} \mathrm{O}$

dehydrogenase activity and urease activity in both before sowing and after harvest of the $\operatorname{crop}(311.11$ and 298.48 $\mu \mathrm{g} \mathrm{TPF} \mathrm{g}^{-1} 24 \mathrm{~h}^{-1}$ and 24.94, $23 \mathrm{~g} \mathrm{NH}_{4} \mathrm{~g}^{-1}$ soil respectively) than mono-cropped finger millet (266.57 and $252.12 \mu \mathrm{g} \mathrm{TPF} \mathrm{g}^{-1} 24 \mathrm{~h}^{-1}$ and $20.18,18.33 \mathrm{~g} \mathrm{NH}_{4} \mathrm{~g}^{-1}$ soil respectively). Application of $100 \%$ recommended dose of fertilizer + organic matter has significantly increased the dehydrogenase activity in finger millet from 143.81, $133.67 \mu \mathrm{g} \mathrm{TPF} \mathrm{g}^{-1} 24 \mathrm{~h}^{-1}$ and 12.47, $11.60 \mathrm{~g} \mathrm{NH}_{4} \mathrm{~g}^{-1}$ (Absolute control) to $441.10,431.13 \mu \mathrm{g}$ TPF $\mathrm{g}^{-1} 24 \mathrm{~h}^{-1} 44.95,43.50 \mathrm{~g} \mathrm{NH}_{4} \mathrm{~g}^{-1}$ in both the cases at 5 per cent probability.

Dehydrogenase activity and urease activity is significantly higher $(0.05 \mathrm{P})$ with rotation including FYM as organic source and $100 \%$ recommended dose of ferti- lizer + organic matter before sowing and after harvest of the crop $\left(628.85,611.46 \mu \mathrm{g}\right.$ TPF $\mathrm{g}^{-1} 24 \mathrm{~h}^{-1}$ and $49.88,49.30 \mathrm{~g} \mathrm{NH}_{4} \mathrm{~g}^{-1}$ respectively) than the monocropped finger millet with no fertilizer but with maize residue@ 5 tonnes ha ${ }^{-1}\left(107.22,101.43 \mu \mathrm{g}\right.$ TPF g ${ }^{-1} 24$ $\mathrm{h}^{-1}$ and 9.29, $9.28 \mathrm{~g} \mathrm{NH}_{4} \mathrm{~g}^{-1}$ respectively). The enzyme activities have been associated with indicators of biogeochemical cycles, degradation of organic matter and soil remediation processes. So, they can determine, together with other physical or chemical properties, the quality of a soil and finally improving the yield of the crop (Gelsomino et al., 2006). Activities of all three enzymes (dehydrogenase, alkaline phosphatase and urease) were positively correlated with biomass N and P with the application of FYM @ 
$4 \mathrm{t}$ per ha. There was significant correlation between soil organic carbon and microbial biomass $\mathrm{C}, \mathrm{N}$ dehydrogenase and urease activities (Manna et al., 2006). The main reason of increased microbial activity was the amount of the organic matter present in the FYM and the nutrient release pattern of the FYM. Some of the interactions were significantly differs but most of them are non-significance.

Acid and alkaline phosphatase activity (g PNP g $^{-1}$ soil): The FYM 10 tonnes $\mathrm{ha}^{-1}$ recorded significantly higher acid and alkaline phosphatase activity before sowing and after harvest of the crop (32.60, 29.96 and $25.6024 .30 \mathrm{~g} \mathrm{PNP} \mathrm{g}^{-1}$ soil, respectively) than with 5 tonnes of maize residue $(19.30,18.31$ and 15.43 and $14.93 \mathrm{gPNP} \mathrm{g}^{-1}$ soil, respectively) at significance level of 5 per cent. Legume rotation system in finger millet has shown significantly higher acid and alkaline phosphatase activity both before sowing and after harvest of the crop (29.57, 28.16 and 24.74, $23.34 \mathrm{~g}$ PNP g${ }^{1}$ soil respectively) than mono-cropped finger millet (22.34, 20.31 and 16.30, 15.89 g PNP g ${ }^{-1}$ soil respectively) at 0.05 probability. The significant difference was noticed in the nutrient source. The highest activity was recorded in $100 \%$ recommended dose of fertilizer + organic matter $(31.54,28.88$ and $32.79,30.68 \mathrm{~g} \mathrm{PNP}$ $\mathrm{g}^{-1} 1$ ) both before sowing and after harvest of the crop. The lowest activity was noticed in the recommended dose of fertilizer $(16.47,15.51$ and $14.31,13.81 \mathrm{~g}$ PNP $\mathrm{g}^{-1}$ soil, respectively).

All the interactions were found to be significant at 5 percent probability. However, with rotation including FYM as organic source and $100 \%$ recommended dose of fertilizer + organic matter has shown the significantly higher acid and alkaline phosphatase activity before sowing and after harvest of the crop (50.67, 47 and $55.10,48.89 \mathrm{~g} \mathrm{PNP} \mathrm{g}^{-1}$ soil, respectively) than the monocropped finger millet with $100 \%$ recommended dose of fertilizer with maize residue @ 5 tonnes ha ${ }^{-1}(11.95,12.77$ and 9.41, 9.04 $\mathrm{g} \mathrm{PNP} \mathrm{g}^{-1}$ soil, respectively).

Yield attributes: Different source of organic matter and rotation system and nutrient sources have significantly influenced the yield and yield parameters at 5 per cent level of significance. Significantly higher ear head length $(7.25 \mathrm{~cm})$, grain weight hill ${ }^{-1}(9.10 \mathrm{~g})$ grain yield $\left(1761 \mathrm{~kg} \mathrm{ha}^{-1}\right)$ and straw yield $\left(2672 \mathrm{~kg} \mathrm{ha}^{-1}\right)$ were noticed in 10 tonnes of FYM ha ${ }^{-1}$ over maize residue 5 tonnes ha ${ }^{-1} 6.66 \mathrm{~cm}, 7.16,1365 \mathrm{~kg} \mathrm{ha}^{-1}$ and $2218 \mathrm{~kg} \mathrm{ha}^{-1}$, respectively. Finger millet grown in mono cropping had significantly lower ear head length $(6.62 \mathrm{~cm})$, grain weight hill $^{-1}(6.87 \mathrm{~g})$, grain yield $\left(1343 \mathrm{~kg} \mathrm{ha}^{-1}\right)$ and straw yield $\left(1990 \mathrm{~kg} \mathrm{ha}^{-1}\right)$ than legume rotation which showed significantly higher yield parameters $\left(7.30 \mathrm{~cm}, 9.38 \mathrm{~g}, 1783 \mathrm{~kg} \mathrm{ha}^{-1}\right.$ and $2900 \mathrm{~kg}$ $\mathrm{ha}^{-1}$ respectively). Among different nutrient source significantly lower ear head length, grain weight hill ${ }^{-1}$ grain yield and straw yield were observed in absolute control $\left(6.44 \mathrm{~cm}, 4.75 \mathrm{~g}, 227 \mathrm{~kg} \mathrm{ha}^{-1}\right.$ and $256 \mathrm{~kg} \mathrm{ha}^{-1}$ respectively). Whereas, $100 \%$ recommended dose of fertilizers + organic matter source has recorded significantly higher (Ear head length (7.92), grain weight hill ${ }^{-1}(11.08)$, grain yield $\left(2741 \mathrm{~kg} \mathrm{ha}^{-1}\right)$ and straw yield $4598 \mathrm{~kg} \mathrm{ha}^{-1}$ respectively). The better performances of FYM over maize residue, legume rotation over mono cropping and recommended fertilizers with organic matter over control were mainly due to balanced and controlled supply of nutrients which have resulted in better growth parameters which have reflected on the improved yield parameters (Table 5) and yield. Ramamoorthy et al. (2009) conducted three year experiment in Coimbatore to know the productivity and profitability of rainfed finger millet through organic farming. The three year study revealed that application of higher levels of FYM @ $12.5 \mathrm{~kg} \mathrm{ha}^{-1}$ gave higher grain $\left(2963 \mathrm{~kg} \mathrm{ha}^{-1}\right)$, higher ear head length $(8.12 \mathrm{~cm})$, test weight $(3.35 \mathrm{~g})$ and straw yield (6030) in finger millet intercropping system. In the interaction effect of organic source $\mathbf{x}$ nutrient source, FYM 10 tonnes ha ${ }^{-1}$ with organic matter $+100 \%$ RDF has recorded significantly higher Ear head length (8.10), grain weight hill ${ }^{1}$ (13.38), grain yield (2846 $\left.\mathrm{kg} \mathrm{ha}^{-1}\right)$ and straw yield $4460 \mathrm{~kg} \mathrm{ha}^{-1}$ than maize residue 5 tonnes $\mathrm{ha}^{-1}+$ absolute control $\left(6.18,5.20,122 \mathrm{~kg} \mathrm{ha}^{-1}\right.$ and $155 \mathrm{~kg} \mathrm{ha}^{-1}$ respectively). Except rotation system $\mathbf{x}$ nutrient sources all other interactions were found to be significant. Under interactions of organic sources $\mathbf{x}$ rotation system $\mathbf{x}$ different nutrient sources, the highest grain weight hill $^{-1}(13.75 \mathrm{~g})$ was noticed in $100 \%$ recommended dose of fertilizer with FYM and legume rotation. However, the least grain weight was noticed in maize residue with absolute control in mono-cropped finger millet (4.90 g).

\section{Conclusion}

The experimental results revealed that application of FYM 10 tones ha $^{-1}$ has given higher grain and straw yield and best physical parameters over the application of maize residue at 5 tonnes ha-1. Among two rotation system legume cereal rotation system will provide the high yield because of improved soil health and microbial activities. Application of organic matter with $100 \%$ RDF has given higher yields as well as good microbial activities and soil health also over the absolute control. Thus, the productivity and profitability of rainfed finger millet could be improved by the application of FYM and compost with better soil health attributes.

\section{REFERENCES}

Anonymous (2013). Annual Report, All India Coordinated Research Project for Dryland Agriculture, University of Agricultural Science, Bangalore, Karnataka, India Pp: 31-36

Birajdar, R. R., Chalwade, P. B., Badole, S. B., Shelage, B. S. and Hangarge, D. S. (2001). Physical chemical properties of vertisolsas affected by fly ash and farm yard manure under sweet potato. J. Soils and Crops, 11(1): 65-68 
Bajpai, R. K., Shrikant, C., Upadhyay, S. K. and Urkurkar, J. S. (2006). Long term studies on soil physico-chemical properties and productivity of rice-wheat system as influenced by integrated nutrient management in inceptisol of Chhattisgarh. J. Indian Soc. Soil Sci., 54 (1): 24-29

Casida, L. E., Klein, D. A. and Santro (1964). Soil dehydrogenase activity. Soil Biol. Biochem., 22(7):190-193

Dhull, S. K., Goyal, S., Kapoor, K. K. and Mundra, M. C. (2005). Crop rotation effects on soil organic matter and soil microbial properties. Indian J. Agric. Res., 39(2): 128-132

Eivazi, F. and Tabatabai, M. A. (1977).Phosphates in soils. Soil Biol. Biochem., 9: 167-172

Gomez, K. A. and Gomez, A. A. (1984). Stastical procedure for agriculltural research. John Wileyand sons, New Delhi p. 680.

Gelsomino, A., Badalucco, L., Ambrosoli, R., Crecchio, C., Puglisi, E. and Meli, S. (2006). Changes in chemical and biological soil properties as induced by anthropogenic disturbance: a case study of agricultural soil under recurrent flooding by waste waters. Soil Biol. Biochem., 38: 2069-2080

Jackson, M. L. (1967). Soil chemical analysis. 2nd ed. Prentice Hall of India Pvt. Ltd, New Delhi p: 498

Manna, M. C., Kundu, S., Singh, M. and Takkar, P. N. (2006). Influence of FYM on dynamics of microbial biomass and its turnover and activity of enzymes under a soybean-wheat system on a typichaplustert. J. Indian Soc. Soil Sci., 44(3): 409-412

Patidar, M. and Mali, A. L. (2014). Effect of farm yard manure, fertility levels and bio-fertilizers on growth, yield and quality of great millet. Indian J. Agron., 49 (2): $117-120$

Ramamoorthy, K., Radhamani, S. and Subbain, P. (2009). Productivity and profitability of rainfed finger millet (Eleusinecoracona (L.) Gaertn) through organic farming. Green Farming, 2(5): 269-271

Yadav, P. V., Chalwade, P. B., Solanke, A S. and Kulkarni, V. K. (2006). Effect of fly ash and FYM on physicochemical properties of vertisols. J. Soils and Crops, 13 (1): 59-64 